\title{
Topografía y Biometría del Sistema Venoso Coronario y de sus tributarias
}

\author{
Topography and Biometry of the Coronary Venous System and its Tributaries \\ "Sousa-Rodrigues, C. F.; "Alcântara, F. S.; Olave, E.
}

SOUSA-RODRIGUES, C. F.; ALCÂNTARA, F. S. \& OLAVE, E. Topografía y biometría del sistema venoso coronario y de sus tributarias. Int. J. Morphol., 23(2):177-184, 2005.

RESUMEN: El seno coronario es la principal terminación de las venas cardiacas, recibiendo toda su sangre, excepto aquella conducida por las venas cardíacas anteriores y venas cardíacas mínimas.Está localizado en la porción posterior del surco coronario, entre el atrio y ventrículo izquierdos. Sus afluentes directos son las vv cardiacas magna (VCM), media (VCMe), parva (VCP), oblicua del atrio izquierdo (VOAI) y posteriores del ventrículo izquierdo (VPVI). Estudiamos el seno coronario y sus afluentes en 26 corazones de cadáveres de individuos brasileños, adultos, de ambos sexos, con el propósito de obtener datos biométricos del mismo. El calibre en la parte central del tercio izquierdo del seno coronario varió entre 4 y $10 \mathrm{~mm}$, con un promedio de 6,34mm; el del tercio medio varió entre 7 y $14 \mathrm{~mm}$, con un promedio de $9,13 \mathrm{~mm}$ y en el tercio derecho, a nivel de su desembocadura, varió entre 5 y $16 \mathrm{~mm}$ con promedio de $9,28 \mathrm{~mm}$. Su longitud se ubicó entre 27 y $54 \mathrm{~mm}$, con un promedio de 40,3 $\mathrm{mm}$.

En todos los corazones la VCM se continuó con el extremo izquierdo del seno coronario. En 22 de ellos (84,6\%), la VOAI desembocó en su tercio izquierdo y en los 4 restantes ésta estuvo ausente. El seno coronario recibió la vena marginal izquierda en 3 casos $(11,5 \%)$. En todos los casos, la VCMe desembocó en el tercio derecho del seno. En 16 casos (61,5\%) alguna VPVI desembocó en su tercio izquierdo.De éstos, hubo una VPVI en 10 corazones (38,5\%), dos VPVI en 4 (15,3\%) y tres VPVI en 2 (7,7\%). En 23 corazones (88,5\%), alguna VPVI lo hizo en su tercio medio y de estos casos, una VPVI terminó en 15 (57,7\%) y dos VPVI en los 8 restantes (30,8\%). En 21 casos $(80,8 \%)$ alguna VPVI terminó en su tercio derecho y de éstos, se observó una VPVI en 14 corazones (53,9\%), dos en $5(19,2 \%)$ y tres en $2(7,7 \%)$.

La biometría del seno coronario es muy variable, así como la disposición de sus tributarias, por lo que esperamos que los datos obtenidos sirvan de soporte morfológico a la anatomía quirúrgica del corazón.

PALABRAS CLAVE: Anatomía; Corazón; Angiología.

\section{INTRODUCCIÓN}

El seno coronario ( $\mathrm{SC}$ ) es la terminación de las venas cardiacas, siendo la principal vía de drenaje venoso del corazón, complementándose con el drenaje de las venas cardíacas anteriores y mínimas (Lockhardt et al. 1965; Moore, 1994). Así, el SC actúa como un vaso colector para la mayoría de las venas superficiales de este órgano (Baroldi \& Giuseppe, 1965). Existe concordancia en la literatura consultada sobre la posición del seno en la parte posterior del surco coronario, entre el atrio y el ventrículo izquierdos, cubierto parcialmente por fibras musculares superficiales del atrio. Adicionalmente, Testut \& Latarjet (1969) relataron que el seno es más marcado en el recién nacido que en el adulto.
Existen discrepancias en torno al inicio del seno, indicándose que es: 1. En el punto de inserción de una válvula rudimentaria (de Vieussens) localizada en la unión entre la vena cardíaca magna (VCM) y el seno coronario, la cual se observa entre un $43 \%$ y $96,5 \%$ de los casos (Baroldi \& Giuseppe). 2. En la unión de la VCM con la vena oblicua del atrio izquierdo (VOAI) o con la vena posterior del ventrículo izquierdo (VPVI), origen señalado por Hollinshead (1966). Además, se han citado dos estructuras cuyas posiciones son equivalentes, por lo que servirían como punto de demarcación para el inicio del seno; tales estructuras corresponden a la válvula mencionada y el punto de desembocadura de la

* Universidade Federal de Alagoas, Brasil.

** Universidad de La Frontera - Temuco - Chile. 
VOAI (Testut \& Latarjet; James, 1961: Baroldi \& Giuseppe). Estos últimos autores dicen que la válvula debe ser considerada como su límite distal, ya que se presenta frecuentemente y además tomar en cuenta la desembocadura de la VOAI sólo en los casos en que la primera se encuentre ausente.

Los principales afluentes del SC corresponden a venas que acompañan a las arterias coronarias (Hamilton, 1983). Sus tributarias directas son las venas cardíacas magna, media (VCMe) y parva (VCP), vena oblicua del atrio izquierdo y venas posteriores del ventrículo izquierdo (Prives et al., 1971; Gardner et al., 1988; Goss, 1989; Lindner, 1989; Woodburne (1984); Williams et al. (1995). Sin embargo, Tandler (1929) y Gardner \& Osburn (1971) no mencionan a las VPVI como tributarias del seno.

Los recientes métodos diagnósticos y terapéuticos que involucran una caterización del seno coronario, han suscitado el interés de investigadores y del nuestro propio, en relación a la biometría y topografía de este vaso, con el propósito de entregar otros datos que complementen los ya existentes y que sin duda, servirán de soporte en cualquier proceso en que se intervenga a través del seno, pudiendo ejecutarlo con mayor seguridad. Entendemos que un mejor conocimiento de su anatomía y de los padrones de afluencia de sus tributarias permitirán tales objetivos.

\section{MATERIAL Y MÉTODO}

El material analizado consistió en 26 corazones de individuos adultos, de ambos sexos, sin anormalidades macroscópicas o historia de alteraciones patológicas cardíacas, fijados en formaldehído al $10 \%$ y disponibles para investigación en los Laboratorios de Anatomía Topográfica del Centro de Ciencias Biológicas de la Universidad Federal de Alagoas y Universidad de Ciencias de la Salud, Maceió, Brasil.

Inicialmente, se delimitó el área a estudiar en la cara diafragmática del corazón; luego, fue realizada la disección del seno coronario y de sus tributarias directas como la vena cardiaca magna, la vena cardiaca media, y cuando existen o drenan directamente en el SC, las venas posteriores del ventrículo izquierdo, la vena cardíaca parva y la vena oblicua del atrio izquierdo.

Tal procedimiento fue acompañado del retiro de una cantidad apreciable de tejido adiposo acumulado bajo el epicardio, buscando una mejor visión de las estructuras estudiadas, verificando su disposición, su trayecto y las relaciones que mantenían entre sí.
Para medir la longitud y calibres en diferentes porciones del seno, se utilizó un paquímetro digital (METRICA). En relación a la topografía del seno, dividimos su extensión en tres segmentos (tercios izquierdo, medio y derecho), de manera que se pudiesen establecer posibles padrones de territorios de afluencia en sus diferentes porciones y registrar los calibres respectivos.

\section{RESULTADOS}

De acuerdo a los registros biométricos, en el estudio del SC, su longitud varió entre $27 \mathrm{~mm}$ y $54 \mathrm{~mm}$, con un promedio de 40,3 $\mathrm{mm}$.

Los calibres obtenidos en diversos niveles de la longitud del seno coronario, fueron los siguientes: en la parte central del tercio izquierdo varió de 4 a $10 \mathrm{~mm}$, con un promedio de $6,34 \mathrm{~mm}$; en el tercio medio varió entre 7 y 14 $\mathrm{mm}$, con un promedio de $9,13 \mathrm{~mm}$ y en el tercio derecho, a nivel de su desembocadura, varió entre 5 y $16 \mathrm{~mm}$, con un promedio de $9,28 \mathrm{~mm}$.

En todos los corazones, la VCM se continuó con el seno coronario en su extremidad izquierda (Fig. 1). No se observó dilatación en la porción terminal de la VCM, a nivel de su desembocadura en el SC.

En 22 casos (84,6\%), la VOAI desembocó en su tercio izquierdo y en los 4 restantes, esta vena no se encontró.

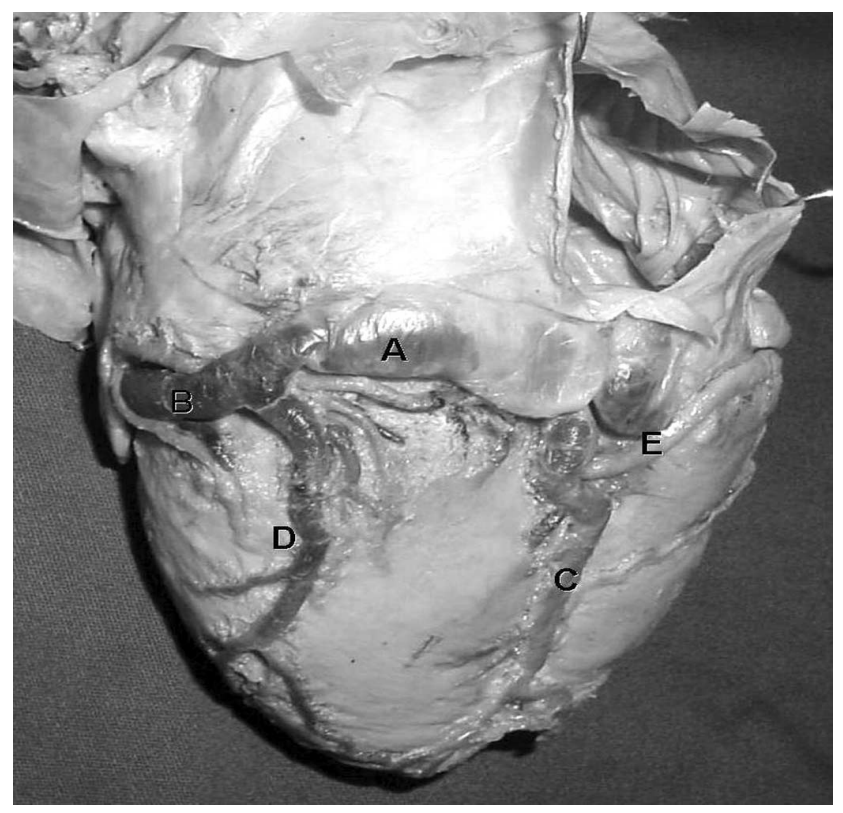

Fig. 1. Vista posterior del corazón (cara diafragmática). A: Seno coronario; B. Vena cardiaca magna; C. Vena cardiaca media; D. Vena posterior del ventrículo izquierdo; E. Arteria coronaria derecha. 
El SC recibió a la vena marginal izquierda en tres casos $(11,5 \%)$, mientras que en los 23 casos restantes $(88,5 \%)$, la misma drenó en la VCM o estaba ausente.

En todos los corazones, la VCMe desembocó en la extremidad derecha del SC (Fig. 2).

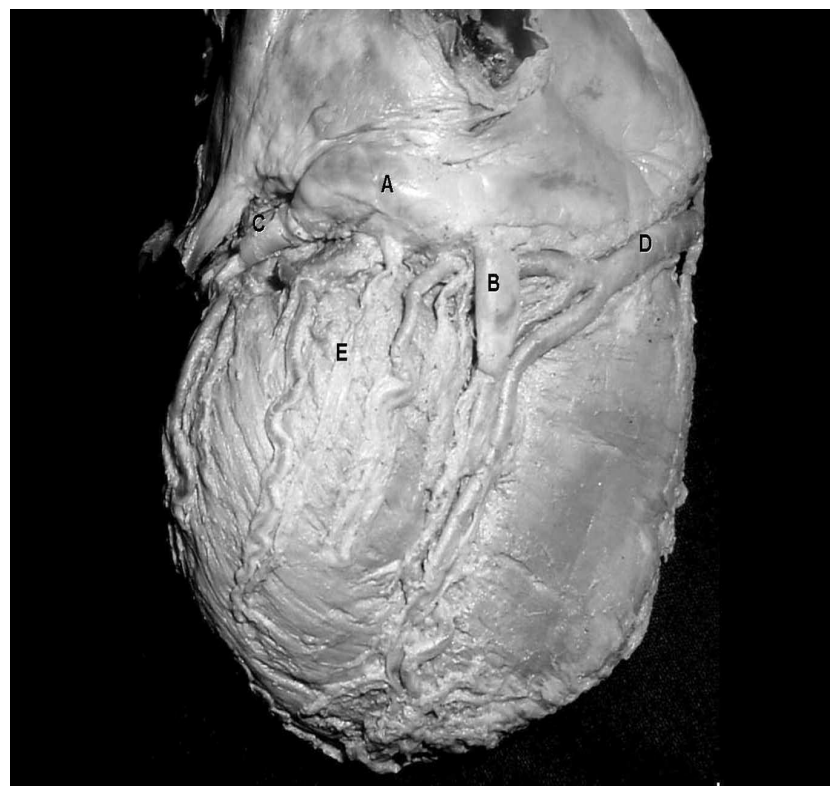

Fig. 2. A. Seno coronario; B; Vena cardiaca media; C. Vena cardiaca magna; D. Arteria coronaria derecha; E. Vena posterior del ventrículo izquierdo.

En la mayoría de los casos, la VPVI se presentó múltiple, siendo observado en uno de los casos $(3,8 \%)$ hasta 6 de éstas desembocando en diferentes puntos, en el SC, en la VCM o en la VCMe. En 16 corazones (61,5\%), alguna VPVI desembocó en el tercio izquierdo del SC; de esos, había una VPVI en 10 casos $(38,5 \%)$; dos VPVI en $4(15,3 \%)$ y 3 VPVI en $2(7,7 \%)$. En 23 corazones $(88,5 \%)$, alguna VPVI desembocó en el tercio medio del SC; de éstos, una VPVI desembocó en 15 casos $(57,7 \%)$ y dos VPVI en 8 (30,8\%). En 21 casos (80,8\%) alguna VPVI desembocó en el tercio derecho del SC, observando una VPVI en 14 de ellos (53,9\%), dos VPVI en 5 $(19,2 \%)$ y tres VPVI en $2(7,7 \%)$. (Fig. 3).

La VCP se presentó en 16 corazones (61,5 $\%$ de los casos) y su desembocadura fue siempre en el SC, indirecta o directamente, no encontrándose ningún caso en que desembocara en el atrio derecho. En 14 casos (53,8\%), la VCP desembocó en la VCMe, a una distancia promedio de $38 \mathrm{~mm}$ del SC; en 2 corazones $(7,7 \%)$, la VCP desembocó directamente en la extremidad derecha del SC.
En general, los afluentes venosos están localizados superficialmente a las arterias con las cuales se relacionan.

Con relación a la disposición de fibras musculares del atrio izquierdo sobre el SC, en la mayoría de los casos, fueron escasas o inexistentes y cuando fueron observadas, éstas tenían una disposición oblicua o transversal.

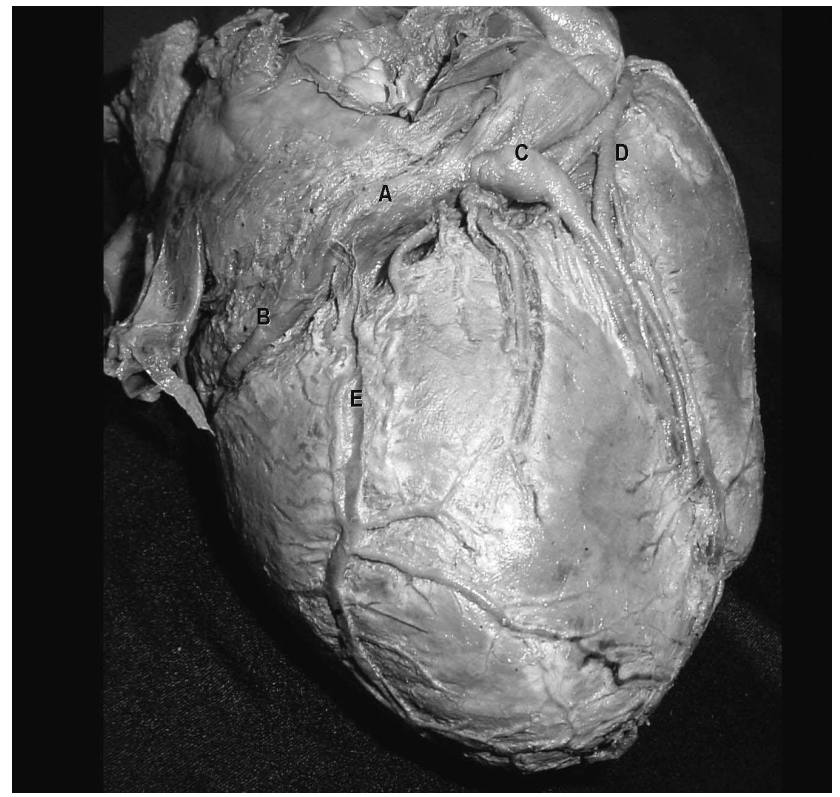

Fig. 3. A. Seno coronario; B. Vena cardiaca magna; C. Vena cardiaca media; D. Arteria coronaria derecha; E Vena posterior del ventrículo izquierdo desembocando en el lado izquierdo del SC; Otras VPVE menores desembocan en el lado derecho del SC.

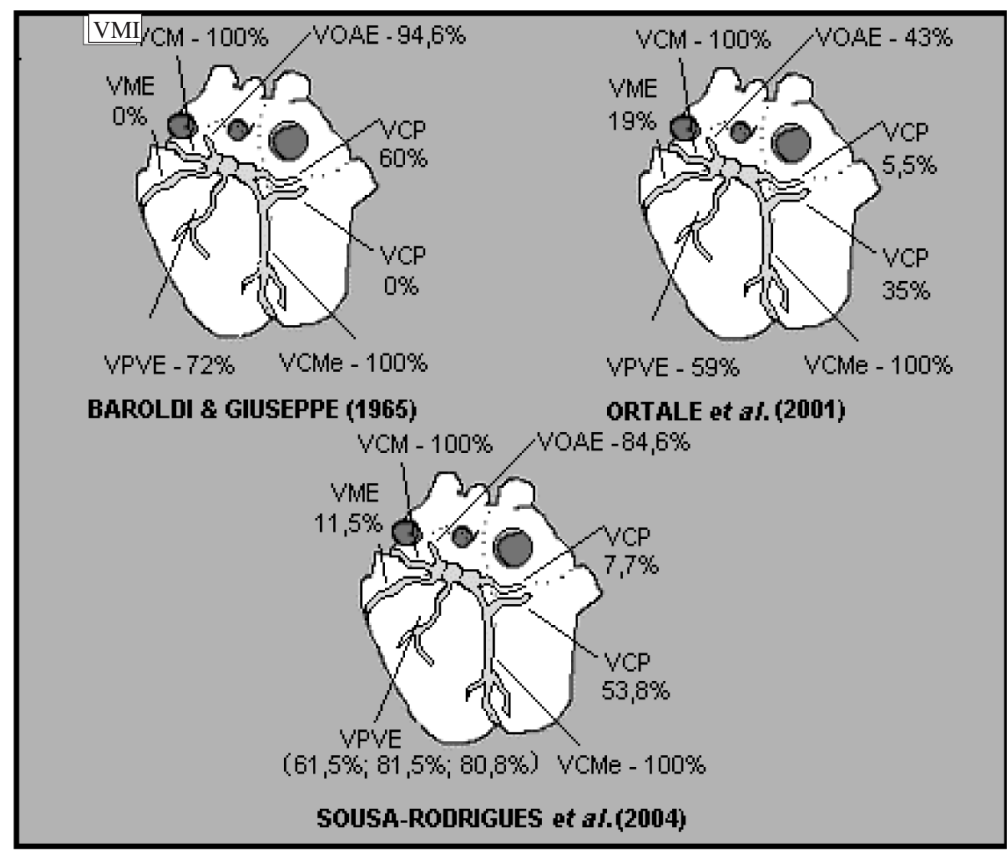

Fig. 4. Representación esquemática que compara la frecuencia del drenaje de diversas venas cardiacas en el seno coronario, según diferentes autores. 


\section{DISCUSIÓN}

El SC es la principal terminación de las venas cardiacas, drenando toda su sangre excepto aquella conducida por las venas cardiacas anteriores y venas cardiacas mínimas. Está localizado en la porción posterior del surco coronario, entre el atrio y ventrículo izquierdos. Con relación a su origen, concordamos con Baroldi \& Giuseppe quienes señalaron que la válvula de Vieussens debe ser considerada como el elemento principal para señalar el límite distal del SC y sólo en los casos en que ésta se encuentra ausente, tomar el punto de llegada de la VOAI como referencia para ello.

No concordamos con Hollinshead, cuando cita que el origen del SC se localiza en la unión de la VCM con la VOAI o con la VPVI, ya que la VOAI puede estar ausente y la VPVI, en la mayoría de los casos, es múltiple, pudiendo desembocar en diferentes puntos en el SC y en otras venas cardiacas, habiendo observado, en una de nuestras muestras hasta seis VPVI alcanzando el SC, la VCM y la VCMe.

Así, considerando que el límite proximal del SC es de consenso en la literatura consultada, el cual corresponde a su desembocadura en el atrioderecho (AD), su límite distal no es de localización tan evidente, encontrando tres estructuras que pueden delimitarlo, cuya posición es en la mayoría de los casos, relativamente equivalente, por lo que se hace necesario establecer una demarcación padronizada para el inicio del SC, la que debería ser de la siguiente forma: 1.- El lugar de la válvula de Vieussens; 2.- El punto de desembocadura de la VOAI; 3.- El límite en que el SC recibe fibras musculares del atrio izquierdo, de acuerdo con los relatos de Alves (1965), Didio (2002) y Tandler. En la mayoría de los casos, por los menos dos de esas demarcaciones son encontradas simultáneamente; sin embargo, basados en nuestra investigación, la cobertura muscular del $\mathrm{SC}$, formada por fibras derivadas del atrio izquierdo, con frecuencia es poco evidente o inexistente.

Tandler afirmó que la disposición de estas fibras musculares sobre el seno coronario es transversal, mientras que DiDio resaltó que es oblicua. En los casos en que constatamos tales fibras, las encontramos dispuestas en cualquiera de los dos padrones.

Otros autores no delimitan el inicio del SC, citando a éste y a la VCM como porciones diferentes de una misma estructura: Testut \& Jacob (1950) se refieren a la VCM y al SC únicamente como "la gran vena coronaria"; Rouvière (1943) y Latarjet \& Ruiz Liard (1983) describieron al SC como una terminación dilatada de la VCM; Testut \&
Latarjet lo denominaron "seno de la vena coronaria mayor", lo que corresponde a la porción terminal dilatada del segmento transversal de la vena coronaria mayor(VCM), localizada en el surco atrioventricular izquierdo.

Los principales afluentes del SC están asociados a las ramas de las arterias coronarias (Hamilton). Sus tributarias directas son las venas VCM, VCMe y VCP, VOAI y venas posteriores del ventrículo izquierdo (Alves, Prives et al., Woodburne, Gardner et al., Goss, Lindner, Williams et al.,). Sin embargo, algunos autores no citan a las últimas como tributarias (Tandler, Gardner \& Osburn).

El SC recibe a la VCM en su extremidad izquierda y a las VCMe y VCP en su extremidad derecha (Moore). Lockhardt et al. y Tandler citaron que estas últimas desembocan juntas en el SC por medio de un tronco común, mientras que Lisboa et al. (1987) y Ortale et al., (2001) afirmaron que la vena marginal derecha puede desembocar en la vCMe.

Según Mochizuki (1933), cerca de su terminación la VCM es, algunas veces, dilatada y esta dilatación corresponde a “Gruber's sinus venae coronariae magnae proprius" y en este caso, ésta ocurriría antes del inicio del SC. Consideramos que la dilatación mencionada no es tal, concordando con Pejkovich \& Bogdanovich (1992). El hecho que a veces, la porción inicial del SC es estrecha en el punto de inserción de la válvula de Vieussens y distal a la desembocadura de la VOAI, podría crear un efecto óptico de dilatación.

Los principales afluentes del SC están asociados a los ramos de las arterias coronarias, concordando con lo descrito por Hamilton. Así, verificamos que la VCM se relaciona, en el surco coronario posterior, a la izquierda del $\mathrm{SC}$, con la rama circunfleja de la arteria coronaria izquier$\mathrm{da}$; la VCMe, con la arteria descendente posterior en el surco interventricular posterior; la VCP (cuando presente), en el surco coronario posterior, con una pequeña porción de la arteria coronaria derecha, antes que ésta ingrese en el surco interventricular posterior. Generalmente, todos estos afluentes venosos se encuentran superficiales a las arterias con las que se relacionan, tal como ha sido descrito por Grant (1948) y Lockhardt et al.. Además, las VPVI, que desembocan en diferentes porciones del margen inferior del SC, se relacionaban con las ramas posteriores del ventrículo izquierdo, ramas de la arteria coronaria izquierda, las que la mayoría de las veces se acompañaban de dos afluentes venosos poco calibrosos. 
Tabla I. Longitud y diámetro del seno coronario según diferentes autores.

$\begin{array}{lcc}\text { Autor } & \text { Longitud }(\mathrm{mm}) & \text { Diámetro }(\mathrm{mm}) \\ \text { Testut } \text { apud } \text { Baroldi \& Giuseppe } & 20-50 & 7-14 \\ \text { Parsonnet } \text { apud } \text { Baroldi \& Giuseppe } & 15-53 \text { (promedio 35) } & 12 \\ \text { Mechanik apud Baroldi \& Giuseppe } & 23-46 & 7-14 \\ \text { Mochizuki } & 5-45 & 5-12 \text { (promedio 8,2) } \\ \text { Aho apud Baroldi \& Giuseppe } & 20-60 \text { (promedio 39) } & 4-15 \text { (promedio 8,7) } \\ \text { Baroldi \& Giuseppe } & 15-55 \text { (promedio 33,6) } & 6-12 \text { (promedio 9,9) } \\ \text { Ortale } \text { et al. } & 27-54 \text { (promedio 40,3) } & \text { Tercio izquierdo 4-10 (6,34); tercio medio 7- } \\ \text { Sousa- Rodrigues } \text { et al. } & & 14(9,13) \text { : tercio derecho 5-16 (9,28) } \\ \text { Rouviere } & 30 & \\ \text { Lockhardt } & 25 & \\ \text { Latarjet-Ruiz Liard } & 30 & \\ \text { Goss } & 22,5 & \\ \text { Moore } & 20 & \\ \text { Williams } \text { et al. } & 20-30 & \\ \end{array}$

Autores como Lockhardt et al. y Tandler han citado que las VCMe y VCP desembocan en el SC por medio de un tronco común, el cual de acuerdo a nuestras observaciones y concordando con Ortale et al. y Lisboa et al., es en verdad, aún la VCMe, ya que la mayoría de las veces, la VCP desemboca en la VCMe y no en el SC, como es comúnmente descrito.

Hollinshead citó que próximo a su terminación, el $\mathrm{SC}$ recibe a las VIP y VCD.

Con relación a la VPVI, Prives et al. y Spalteholz (1967) comentaron que éstas pueden desembocar en el SC y/o en la VCM. Por otra parte, Pejkovich \& Bogdanovich señalaron que mientras más corto sea el SC, menor número de VPVI desembocarán en él y en ese caso, lo harán en la VCM, sin embargo, estos mismos autores relataron que las VPVI drenaron en el SC en $91 \%$ de los casos por ellos estudiados, en el SC y en la VCM en $7 \%$ y únicamente en la $\mathrm{VCM}$ en $2 \%$.

Respecto a las mismas venas, Melo et al. (1998) y Ortale et al. citaron que su número varía de 1 a 4 . Los primeros encontraron a esta venas desembocando en el SC en $59 \%$, en el SC y VCM en $30 \%$ y solamente en la VCM en $11 \%$, mientras que los segundos relataron que las VPVI están generalmente representadas por una vena bastante desarrollada en relación a las demás.

Sobre la VCP, James describió que desemboca en el $\mathrm{SC}$, pero puede hacerlo también en el atrio derecho y en la VCMe. Lüdinghausen (1987) la encontró presente en $36 \%$ y Lisboa et al. en 30,4\% de los casos, indicando además que ella drenaba en el SC en $8,7 \%$ y en el $21,7 \%$ restante lo hacía en la VCMe. Ya, Ortale et al. encontraron a la VCP en $54 \%$ y de este porcentaje, $35 \%$ desembocó en la VCMe,
$5,5 \%$ en el SC y en el 13,5 \% drenó en el AD, ya que pertenecía al grupo de venas anteriores.

En relación a las medidas biométricas del seno coronario, la literatura es tan divergente que, para una mejor comparación, presentamos algunos autores y sus resultados en la Tabla I.

Es evidente que diversos factores tienen influencia en la determinación del calibre y longitud de los vasos cardiacos, desde el biotipo y condicionamiento físico de los individuos, que influirán en el tamaño del corazón y en las variaciones entre las anastomosis de sus vasos, sin contar patologías como las dilataciones e hipertrofias ventriculares.

Comprendiendo ese aspecto y observando la tabla $\mathrm{N}^{\circ}$ 1 , se hace fácil entender que uno de los puntos más controvertidos en el estudio del SC es su biometría. Otros factores también pueden explicar tal situación. Una eventual dilatación proximal de la VCMe en su desembocadura, puede dar una falsa impresión de continuación del SC cuando éste ya desembocó en el atrio derecho. En otros casos, la VCMe parece desembocar en el SC, en circunstancias que lo hace en el atrio derecho por medio de un ostio independiente, que se localiza lateralmente al ostio del SC, disposición encontrada por Lüdinghausen en un $13 \%$ de los casos.

En los casos en que la VOAI estuvo ausente (4 / $15,4 \%)$, se hizo dificil la demarcación del inicio del SC, lo que pudo ser realizado considerando el punto de implantación de la válvula en el inicio del SC (de Vieussens). Por otra parte, es claro que mientras menos venas desemboquen en el SC será menor su calibre y posiblemente su longitud, concordando con las descripciones de Pejkovich \& Bogdanovich. Algunos autores podrían no considerar que las VPVI y la vena marginal izquierda pueden desembocar 
en diferentes puntos de la VCM y/o en el SC, llevándolos a incurrir en un error en cuanto a la longitud de este último.

En el estudio de Baroldi \& Giuseppe, la frecuencia de la VPVI se refiere sólo a aquellas que desembocaron únicamente en el SC; la VCM y la VCMe fueron encontradas terminando en el SC en todos los corazones; en relación a la vena marginal izquierda (VMI), ninguna drenó en el SC, haciéndolo únicamente en la VCM; la VOAI fue encontrada en $94,6 \%$, mientras que los autores no hacen mención a la desembocadura de la VCP en la VCMe; en 60 \%, la VCP drenó en el SC.

Según el relató de Ortale et al. la VCM y la VCMe fueron encontradas desembocando en el SC en todas sus muestras; la VMI drenó en el tercio izquierdo del SC en sólo $19 \%$; con objetivo comparativo se consideraron sólo las VPVI que terminaron en el SC, observando tal disposición en 59\%. La VOAI estuvo presente en 43\%; la VCP terminó en la VCMe en $35 \%$ y directamente en el SC en sólo 5,5\%.

En nuestro estudio, así como en la descripción de los autores citados previamente, la VCM y la VCMe fueron observadas en el $100 \%$ de los casos, desembocando en las extremidades izquierda y derecha del SC, respectivamente. La VMI alcanzó el SC en sólo 11,5\%, curvándose abruptamente hacia la derecha para alcanzarlo en su tercio izquierdo.

La VOAI, cuando presente, siempre desembocó en el tercio izquierdo del SC; Las VPVI, son variables en número y drenan en diferentes partes del SC, aunque más frecuentemente en el tercio medio del SC. La VCP estuvo presente en 61,5\% y de éstos, 53,8\% drenaron en la VCMe y en 7,7\% directamente en el SC, concordando con Ortale et al. y Lisboa et al. Sin embargo, se describe comúnmente que la VCP desemboca en la extremidad derecha del SC.

En relación a la desembocadura del $\mathrm{SC}$ es de pleno consenso que drena en el AD, entre los ostios de la vena cava inferior y atrioventricular derecho. Abramson \& Dobrin (1984) señalaron además, que el ostio se encuentra dispuesto lateralmente en el septo interatrial y Latarjet \& Ruiz Liard, encima y atrás del nodo atrioventricular.

La válvula de la desembocadura del SC fue citada como válvula del seno coronario por DiDio, Goss, Moore, Gardner et al. y Woodburne, como "válvula de Thebesio" por Lockhardt et al.,Testut \& Latarjet, Williams et al. y Alves. Por su parte, Williams et al. la describieron como una válvula semilunar, pequeña, incompleta, de una cúspide, variable en tamaño y forma, que se localiza en el margen derecho de su ostio. Según Moore, esa válvula es un vestigio de la válvula del seno venoso (una parte del corazón embrionario que parece no tener función alguna después del nacimiento), sin embargo Gardner \& Osburn citaron que la misma orienta la corriente sanguínea hacia el ostio atrioventricular derecho.

Basados en lo anterior, podemos señalar que las descripciones sobre el tema divergen en aspectos importantes, tanto en el punto de origen del SC, como también respecto a sus afluentes directos o indirectos, lo que hace difícil padronizar las disposiciones encontradas. Sin embargo, los antecedentes obtenidos en nuestra serie complementarán el conocimiento de la anatomía vascular del corazón.

SOUSA-RODRIGUES, C. F.; ALCÂNTARA, F. S. \& OLAVE, E. Topography and biometry of the coronary venous system and its tributaries. Int. J. Morphol., 23(2):177-184, 2005.

SUMMARY:The coronary sinus (SC) is the main end of the cardiac veins, being the principal vein of the heart, draining all its blood, except that driven by the anterior cardiac veins and the minimum cardiac veins. Its location is well-defined in the posterior portion of the coronary sulcus between left atrium and ventricle. Its direct tributaries are the cardiac veins: magna (VCM), media (VCMe), parva (VCP), oblique of the left atrium (VOAE), posterior veins of the left ventricle (VPVE).

The diameter in the middle of the distal third of the coronary sinus ranged from 4 to $10 \mathrm{~mm}$ (mean 6,34 mm); about its medium third, such value ranged from 7 to $14 \mathrm{~mm}$ (mean $9,13 \mathrm{~mm}$ ) and in its proximal third, its diameter ranged from 5 to $16 \mathrm{~mm}(\mathrm{mean} 9,28$ $\mathrm{mm}$ ). Its length ranged from 27 to $54 \mathrm{~mm}$ (mean 40,3 $\mathrm{mm}$ ).

In 26 hearts (100\% of the cases), the VCM continued into the SC in its left side; in 22 cases $(84,6 \%)$, the VOAE flowed into its left third; in the remaining $4(15,4 \%)$, it was absent; The SC received the left marginal vein in 3 hearts $(11,5 \%)$. In 26 cases $(100 \%)$, the VCMe discharged in the right extremity of the SC; in 16 hearts (61,5\%), some VPVE drained into the left third of the SC, in these there was 1 VPVE in $10(38,5 \%), 2$ VPVE in $4(15,3 \%)$ and 3 VPVE in $2(7,7 \%)$. In 23 hearts $(88,5 \%)$, some VPVE discharged in the middle third of the SC; in these, there was 1 VPVE in 15 cases (57,7\%) and 2 VPVE in 8 (30,8\%). In 21 hearts (80,8\%), some VPVE drained into the right third of the SC, in these, there was 1 VPVE in 14 (53,9\%), 2 VPVE in 5 (19,2\%), 3 VPVE in $2(7,7 \%)$.

One of the most polemic aspects in the study of the coronary sinus is its own biometry, contributing to this divergence the absence of a standart location to its beginning and a pattern on tributaries disposition.

KEY WORDS: Anatomy; Heart; Angiology. 


\section{REFERENCIAS BIBLIOGRÁFICAS}

Abramson, D. I. \& Dobrin, P.B. Blood Vessels and Lymphatics in Organ Systems, Academis Press, 1984.

Alves, E. Anatomia Descritiva. Rio de Janeiro, Atheneu, 1965.

Baroldi, G. \& Giuseppe, S. Coronary Circulation in the normal and the pathologic heart. Washington, Armed Forces Institute of Pathology, 1965.

Didio, L. J. A. Tratado de Anatomia Sistêmica Aplicada. $2^{\circ}$ ed. São Paulo, Atheneu, 2002. V.2.

Gardner, W. D. \& Osburn, W. A. Anatomia Humana. Estrutura do Corpo. São Paulo, Atheneu, 1971.

Gardner, E.; Gray, D. J.; \& O’Rahilly, R. Anatomia. 4. ed. Rio de Janeiro, Guanabara-Koogan, 1988.

Goss, C. M. Gray Anatomia. 29. ed. Rio de Janeiro, Guanabara-Koogan, 1989.

Grant, J. C. B. A Method of Anatomy Descriptive and Deductive. 40. ed. Baltimore, Williams and Wilkins Company, 1948.

Hamilton, W. J. Tratado de Anatomia Humana. 2. ed. São Paulo, Interamericana 1983.

Hollinshead, W. H. Anatomía Humana. Rosario, La Médica, 1966.

James, T. N. Anatomy of the Coronary Arteries. 1. ed. New York, Medical Division of Harper \& Brothers, 1961.

Latarjet, M. \& Ruiz Liard, A. Anatomia Humana. 2. ed. Buenos Aires, Panamericana, 1983. V. 2.

Lindner, H.H. Clinical Anatomy. California, Appleton \& Lange, 1989.

Lisboa, L. A. F. et al. Estudo anatômico quanto à presença e drenagem das veias cardíacas humanas (Média, Parva e Marginal Direita). Arquivo Médico do Hospital da Faculdade de Ciências Médicas da Santa Casa de São Paulo, 7(29):37-42, 1987.

Lockhardt, R. D.; Hamilton, G. F. \& Fyfe, F.W. Anatomía Humana. México, Interamericana, 1965.
Lüdinghausen, M.V. Clinical Anatomy of the Cardiac Veins. Surg. Radiol. Anat., 9:159-68, 1987.

Melo, W. D. S; Prudencio, L. A. R; Kusnir, C. E; Pereira, A. L. N; Marques, V;.

Mochizuki, S. Vv. Cordis. In Adachi, B. Das Venensystem der Japaner. Tokyo, Kenkyusha, 1933.

Moore, K. L. Anatomia Orientada para a Clínica. $3^{\circ}$ ed. Rio de Janeiro, Guanabara-Koogan, 1994.

Ortale J. R.; Gabriel, E. A.; Iost, C.; Marquez, C. The Anatomy of the Coronary Sinus and its Tributaries. Surg. Radiol. Anat., 23:15-21, 2001.

Pejkovich, B. \& Bogdanovich, D. The Great Cardiac Vein. Surg. Radiol. Anat. 14:23-8, 1992.

Prives, M.; Lisenkov, N. \& Bushkovich, V. Anatomia Humana. Mir, Moscu, 1971.

Rouvière, H. Anatomie Humaine. Paris, Masson et Cie, 1943.

Spalteholz, W. Atlas de Anatomía Humana. Barcelona, Editorial Labor, 1967. V. 2.

Tandler, J. Tratado de Anatomía Sistemática. $3^{\circ}$ ed. Barcelona, Salvat, 1929.

Testut, L. \& Jacob, O. Tratado de Anatomía Topográfica con aplicaciones médicoquirúrgicas. $8^{\circ}$ ed. Barcelona, Salvat, 1950.

Testut, L. \& Latarjet, A. Compendio de Anatomía Descriptiva. $22^{\circ}$ ed. Barcelona, Salvat, 1969.

Vieira, M. C; Paola, A. A. V. Anatomia Angiográfica do Sistema Venoso Coronário - Aplicações em Eletrofisiologia Clínica. Arq. Bras.Cardiol., 70:6, 1998.

Williams, P. L.;Warwick, R.; Dyson, M. \& Bannister, L. H. Gray Anatomia . $37^{\circ}$ ed. Rio de Janeiro, GuanabaraKoogan, 1995.

Woodburne, R. T. Anatomia Humana. $6^{\circ} \mathrm{ed}$. Rio de Janeiro, Guanabara-Koogan, 1984. 
SOUSA-RODRIGUES, C. F.; ALCÂNTARA, F. S. \& OLAVE, E.

Direçción para correspondencia:

Prof. Dr. Célio Fernando de Sousa Rodrigues

Disciplina de Anatomia Topográfica

Centro de Cièncias Biologicas - UFFAL

Praça Afrânio Jorge, $s / n^{\circ}$ Prado

CEP:57.010-060

Maceió, Alagoas

BRASIL

Email: cfsr2@aol.com ufal0128@fapeal.br
Recibido : 11-12-2004

Aceptado: 10-04-2005 